The Astrophysical JournaL, 307:415-425, 1986 August 15

(C) 1986. The American Astronomical Society. All rights reserved. Printed in U.S.A.

\title{
SELF-REGULATED COOLING FLOWS IN ELLIPTICAL GALAXIES AND IN CLUSTER CORES: IS EXCLUSIVELY LOW MASS STAR FORMATION REALLY NECESSARY?
}

\author{
JOSEPH SILK \\ Department of Astronomy, University of California, Berkeley \\ S. DJORGOVSKI ${ }^{1}$ \\ Department of Astronomy, University of California, Berkeley, and Harvard-Smithsonian Center for Astrophysics \\ Rosemary F. G. WySE. \\ Department of Astronomy, University of California, Berkeley, and Space Telescope Science Institute \\ AND \\ Gustavo Bruzual A. \\ Centro de Investigaciones de Astronomia, Mérida, Venezuela \\ Received 1985 July 8; accepted 1985 September 18
}

\begin{abstract}
A self-consistent treatment of the heating by supernovae associated with star formation in a spherically symmetric cooling flow in a cluster core or elliptical galaxy is presented. An initial stellar mass function similar to that in the solar neighborhood is adopted. Inferred star formation rates, within the cooling regiontypically the inner $100 \mathrm{kpc}$ around dominant galaxies at the centers of cooling flows in XD clusters-are reduced by about a factor of 2, relative to rates inferred when the heat input from star formation is ignored. Truncated initial mass functions (IMFs) are also considered, in which massive star formation is suppressed in accordance with previous treatments, and colors are predicted for star formation in cooling flows associated with central dominant elliptical galaxies in clusters and with isolated elliptical galaxies surrounded by gaseous coronae. The low inferred cooling flow rates around isolated elliptical galaxies are found to be insensitive to the upper mass cutoff in the IMF, provided that the upper mass cutoff exceeds $2 M_{\odot}$. Comparison with observed colors favors a cutoff in the IMF above $1 M_{\odot}$ in at least two well-studied cluster cooling flows, but a normal IMF cannot be excluded definitively. Our models for NGC 1275 support a young ( $\$ 3$ Gyr) cooling flow. As for the isolated elliptical galaxies, the spread in colors is consistent with a normal IMF. A definitive test of the IMF arising via star formation in cooling flows requires either ultraviolet spectral data or supernova searches in the cooling flow-centered galaxies.
\end{abstract}

Subject headings: galaxies: clustering - galaxies: stellar content - intergalactic medium stars: formation - stars: supernovae

\section{INTRODUCTION}

Analysis of the X-ray characteristics of galaxy clusters has led to the discovery of cooling flows (Fabian, Nulsen, and Canizares 1984, and references cited therein). A recent analysis (Stewart, et al. 1984a) concluded that mass flow rates of between 2 and $400 M_{\odot} \mathrm{yr}^{-1}$ occurred in a substantial fraction of XD clusters, defined to be those compact X-ray clusters centered on a single optically dominant galaxy. Cooling flows are recognized by evaluation of the central cooling time scale, derived from the X-ray profile, found to be less than a Hubble time. To a first approximation, the mass-flow rate may then be taken to equal the ratio of the gas mass within a spherical shell to the local cooling time. The gas is assumed to accrete subsonically in a pressure-driven flow.

If the flows are steady, the only plausible sink for the infalling gas is star formation. Practically all observations of star formation and spectral synthesis of other galaxies are consistent with a universal IMF (Scalo 1985). Yet it has been argued that the colors of the central galaxies preclude the occurrence of star formation at this inferred rate with an initial mass function (IMF) similar to that in the solar neighborhood. Fabian, Nulsen, and Canizares (1982) and Sarazin and O'Connell

\footnotetext{
${ }^{1}$ Harvard Junior Fellow.
}

(1983) have therefore proposed that predominantly low mass stars are being formed in the central dominant galaxies by the accreting gas. This argument has been extended to the gaseous halos recently discovered around isolated ellipticals. Now, while the pressure in these flows exceeds that in the diffuse interstellar medium clouds by orders of magnitude, with a corresponding reduction in the isothermal Jeans mass, it is by no means straightforward to draw any inference about the ensuing effect on star formation. The actual sites of galactic star formation, molecular cloud cores, have similar pressures. Moreover, one can argue on theoretical grounds for excessive pressure either to enhance low mass (Jura 1977) or massive (Klein, Sanford, and Whitaker 1983; Silk 1985) star formation; one recent observational result indicates that a burst of $\mathrm{OB}$ star formation was triggered in a high pressure environment (van Breugel et al. 1985). This only illustrates the lack of rigor in the theoretical argument favoring low-mass stars.

We shall show here that the observational objections against ongoing star formation with a solar neighborhood IMF, notably involving broad-band $U B V$ colors, are also not very rigorous. Allowing star formation with a normal IMF extending to high mass forces one to consider feedback into the cooling flow due to energy injection from supernovae. The high inferred rate of mass infall implies enough energy input 
via supernovae to modify severely the accretion flow. We will show that this modification is most effective within the innermost regions $(\sim 10 \mathrm{kpc})$ of the flow, while the overall mass infall rate is reduced by about a factor of 2 . In addition, there are uncertainties due to reddening and the enhanced metallicity of the central galaxy affecting the predicted colors: we shall show that the observed colors of the luminous central galaxies are consistent with star formation rates as high as $10 M_{\odot} \mathrm{yr}^{-1}\left(10^{11} L_{\odot}\right)^{-1}$.

Indirect evidence for heating of intracluster gas comes from comparison of the X-ray surface brightness profiles with galaxy counts. Jones and Forman (1984) find that the energy per unit mass in galaxies relative to that in the gas is equal to 0.66 $( \pm 0.1)$ for isothermal model fits. Polytropic models tend to give better fits to the X-ray spectral data, and are found to result in even higher gas temperatures (Mushotzky 1984). An additional indicator of the occurrence of gas heating is that while all clusters with inferred cooling flows have central excesses in the X-ray surface brightness relative to hydrostatic model fits, there are several clusters with X-ray excesses whose central cooling times exceed a Hubble time scale (Jones and Forman 1984). This seems to provide "smoking gun" evidence for heating as well as cooling. Finally, we note that the cooling flows are often associated with strong nonthermal radio sources. Well-studied examples such as NGC 1275 reveal the presence of an extended radio source of comparable luminosity to the compact nuclear radio source. Diffuse nonthermal emission in our own Galaxy is attributed to many very old supernova remnants, which are efficient relativistic particle accelerators and have massive stellar precursors, and a similar signature may be present in the intracluster gas. Indeed, Tucker and Rosner (1983) have suggested that nonthermal heating of the relativistic electrons in the radio lobes around M87 may support the halo gas hydrostatically, although there are problems with the details of this heating mechanism (Fabian, Nulsen, and Canizares 1984). The excellent correlation between nonthermal radio continuum flux and IRAS flux for a sample of late type, actively star forming galaxies (Helou, Soifer, and Rowan-Robinson 1985) suggests that there is massive star formation associated with the radio sources.

Indications that the simple $\dot{M}$ estimates neglecting heating are overestimates come from spectroscopic and photometric comparisons between normal elliptical galaxies, and those at the centers of cooling flows. Specifically, the CO line index work of Gilmore and Arnaud (1986), which is sensitive to the dwarf-to-giant ratio of the stellar population, has revealed no apparent difference in $M / L$ between galaxies with $\dot{M}$ of $60 M_{\odot}$ $\mathrm{yr}^{-1}$ and normal ellipticals with no cooling flows. Their analysis is most sensitive to stars of mass in the range $0.7-0.3$ $M_{\odot}$, just the low-mass range originally favored for cooling flows (Fabian, Nulsen, and Canizares 1982), and suggests that if any IMF variations are allowed, they must be so extreme as to produce an upturn in the IMF at $\lesssim 0.1 M_{\odot}$. Romanishin (1986) has made a near-infrared and optical photometric survey of galaxies at the centers of cooling flows, and he again finds no apparent difference (at the $2 \sigma$ level) in $V-K$ color between galaxies with $0 \lesssim \dot{M} \lesssim 100 M_{\odot} \mathrm{yr}^{-1}$. He rules out long-lived $\left(\sim 10^{10}\right)$ cooling flows at the inferred $\dot{M}$, but cannot rule out flows and star formation over a few times $10^{9} \mathrm{yr}$. Rose (1985) has considered the $\sim 4000 \AA$ light from the nuclei of a sample of ellipticals in some detail; he finds evidence for an intermediate age ( $\sim$ few Gyr) component in all the galaxies, with no difference between NGC 4472, purported to have a substantial cooling flow, and the remainder of the sample.
In what follows, we focus on a rather specific heating mechanism. The presence of a considerable mass of iron (up to $\sim 12$ $M_{\odot}$ ) in rich clusters (Rothenflug and Arnaud 1985) strongly suggests that supernova mass injection into the intracluster gas has occurred. That the associated energy injection may suffice to drive an intracluster wind now seems rather unlikely (Yahil and Ostriker 1973; Sarazin 1985), but the heating of the intracluster gas by supernovae is surely important. A discussion of cooling flows heated by supernovae is presented in $\S$ II. Comparison with observations of clusters is made in $\S$ III, and present models of color and luminosity evolution for accreting galaxies in $\S$ IV. A final section summarizes our main results.

\section{COOLING FLOWS INCORPORATING SUPERNOVA HEATING}

To investigate the effects of supernova heating on cooling flows, we proceed as follows. Let the supernova rate associated with the mass inflow be denoted by $v$ per year per unit star formation rate (in $M_{\odot} \mathrm{yr}^{-1}$ ). We consider only supernovae of Type I on the grounds that these occur in all galaxies, from star forming irregulars to ellipticals, where we believe that mass flows under similar conditions to those studied here may be responsible for star formation. A class of SNI may be associated with massive ( $\gtrsim 4-6 M_{\odot}$ ) precursors (Oemler and Tinsley 1979; Fillipenko and Sargent 1985), and we shall utilize these in the models discussed below to provide a specific example of supernova heating. The ratio of the supernova rate averaged over a Miller-Scalo (1979) IMF to the star formation rate is

$$
v \approx\left(20 M_{\odot}\right)^{-1} .
$$

Inclusion of SNII, which have massive $\left(\gtrsim 10 M_{\odot}\right)$ stellar precursors, will augment this rate. If $E_{\mathrm{SN}}$ denotes the energy input per supernova explosion (and $\dot{M}_{\text {gas }}$ denotes the rate of spherically symmetric inflow in $M_{\odot} \mathrm{yr}^{-1}$, assumed to be entirely fed into star formation) the heat input per unit mass by supernovae is (see White and Chevalier 1983)

$$
\lambda=v E_{\mathrm{SN}}=2 \times 10^{16} E_{51} \operatorname{ergs~}^{-1},
$$

where

$$
E_{51}=E_{\mathrm{SN}} / 10^{51} \mathrm{ergs} \text {. }
$$

The value of $\lambda$ is proportional to the supernova rate per unit rate of star formation and depends on the adopted form of the IMF (and on $E_{\mathrm{SN}}$ ).

We presume that the radial gradient in the mass flow rate, to be derived self-consistently below, yields the star formation rate as a function of distance from the cluster center. This means that, provided flow velocities are sufficiently subsonic, the radial dependences of heating and cooling inevitably coincide, which is desirable if the flow is to remain steady. A mass flow rate independent of radius would imply that star formation occurs only in the very central regions where the solution must break down; however, the observed X-ray emission profiles require models with no heating, in which the mass flow rate declines toward the cluster center (Fabian, Nulsen, and Canizares 1984). As will be seen below, heating accentuates this effect, leading to a reduced rate of star formation at radii where the cooling time is much less than a Hubble time.

We first assume that the star formation rate $\dot{\rho}_{*}=-\dot{\rho}$. We let $\dot{\rho}_{*}(r)$ describe the sinks (or sources) of the flow, write the supernova heating rate as $\lambda \rho_{*}$, and the cooling rate as $\Lambda(T) \rho^{2}$. The interpretation of $\dot{\rho}_{*}$ as star formation rate requires the gas to drop out of the flow and form stars at the same radius. This approximation is in accord with the subsonic nature of the flow; we shall discuss the effects of finite flow velocity in $\S \mathrm{V}$ 
below. It will be assumed that, while the flow is thermally unstable, the bulk of the cooling occurs in the hot X-ray emitting gas. A spherically symmetric, steady subsonic gas flow can then be described by the following equations, where terms of second order in the ratio of flow velocity $v$ to sound speed have been neglected:

Energy:

$$
\nabla \cdot \rho v(H+\phi)=-\rho^{2} \Lambda+\lambda \dot{\rho}_{*}+\dot{\rho} \phi .
$$

Momentum:

$$
\frac{d p}{d r}=-\rho \nabla \phi
$$

Continuity:

$$
\dot{\rho}_{*}=-\nabla \cdot(\rho v)
$$

Here the enthalpy

$$
H=\left(\frac{\gamma}{\gamma-1}\right) \frac{p}{\rho}=\frac{5}{2} \frac{k T}{\mu m_{\mathrm{H}}} ;
$$

$\gamma$ is the adiabatic index for a monatomic gas, and $\mu$ is the mean molecular weight. We further specialize by assuming spherical symmetry, writing the inward flow velocity $\boldsymbol{v}=-(v, 0,0)$ and the total star formation rate within the radius $r$ as $\dot{M}_{*}(r)=$ $\int_{0}^{r} 4 \pi \dot{\rho}_{*} r^{2} d r$. The energy equation reduces to

$$
-\dot{\rho}_{*} H+\frac{2}{5} \frac{\dot{M}_{*}}{4 \pi r^{3}} H \frac{d \ln }{d \ln r}\left(\frac{T^{3 / 2}}{\rho}\right)=\lambda \dot{\rho}_{*}-\Lambda \rho^{2},
$$

where we explicitly allow for mass sinks (or sources) and a radial dependence of $\dot{M}_{*}$. Radial dependences of the various variables are introduced by writing

$$
\begin{gathered}
\delta=d \ln \phi / d \ln r ; \quad \beta=d \ln \rho / d \ln r ; \\
\xi=d \ln \dot{\rho}_{*} / d \ln r ; \quad \eta=d \ln T / d \ln r .
\end{gathered}
$$

The gravitational potential $\phi$ of the galaxy cluster core is presumed to be known. The density and temperature profiles of the X-ray emitting gas can be derived from high-resolution $\mathrm{X}$-ray mapping, and we shall derive the flow rate gradient.

The energy equation now yields

$$
\dot{\rho}_{*}=\frac{\Lambda \rho^{2}}{\lambda+\alpha H},
$$

where

$$
\alpha=1+\frac{2 \beta-3 \eta}{5(\xi+3)} .
$$

For a solution to be valid over some finite range in radius, the radial dependences of all terms must be similar. Hence a solution with heating is given by

$$
\xi=2 \beta, \quad \eta=0 \text {. }
$$

This describes an isothermal flow, with a star formation that is proportional to the square of the local gas density, and we obtain

$$
\alpha=\left(1+\frac{4}{5} \beta\right) /\left(1+\frac{2}{3} \beta\right)
$$

In well-studied cooling flows, such as those around M87 (Stewart et al. 1984b) and NGC 1275 (Fabian et al. 1981), the density dependence is often modeled to fit $\beta \approx-1$ : for $\beta=-1( \pm 0.2)$ we have $\alpha=0.6\left({ }_{-0.4}^{+0.2}\right)$. In the absence of a heating term, the solution is not restricted to being isothermal, and a variety of solutions are possible for different assumptions about the gravitational potential. These include a constant pressure flow, when the gravitational acceleration can be neglected, and we have taken $\Lambda(T) \propto T^{a}$, so that

$$
\dot{\rho}_{*} \propto \rho^{3-a} .
$$

Over $2 \times 10^{6}-3 \times 10^{7} \mathrm{~K}, \Lambda \approx$ constant, so that $a \approx 0$. In a constant pressure flow, one therefore expects $\dot{M}_{*} \approx$ constant if $\beta \approx-1$.

In fact, modeling of cooling flows demonstrates that constant pressure is not a good approximation. This can be seen directly from the observed density and temperature profiles: typically, $\beta \approx-1$ and $0 \leq \eta \leq 0.5$. For example, if gravity is neglected, the modeling of the flow centered on NGC 1275, where $\beta \approx-1, \eta \approx 0.5$ (Fabian et al. 1981), yields $\dot{\rho}_{*} \propto \Lambda \rho^{2} / T$ or (with $a=\frac{1}{2}$ ) $M_{*} \propto r^{0.75}$ in a purely cooling flow. However, whdn heating is dominant, the radial dependence asymptotically steepens to $\dot{\rho}_{*} \propto \rho^{2} \Lambda / \lambda$ or $\dot{M}_{*} \propto r^{5 / 4}$. This is similar to the radial flow dependence found by deconvolving the observed X-ray emissivity into radial shells (Fabian, Nulsen, and Canizares 1984), perhaps providing evidence that heating is playing a role. Similarly for the somewhat softer source around $\mathrm{M} 87, \beta=-1.2, \eta=0.8$, and $a \approx 0$, so that $\dot{M}_{*} \propto r^{0.6}$ in a heating flow, again in agreement with the radial deconvolution results (Stewart et al. 1984b); compare this with a pure cooling flow, in which $\dot{M}_{*} \propto r^{0.2}$. Thus the X-ray data appear to support a heating model. Moreover, the quadratic dependence of star formation rate on gas density inferred for the heating model is similar to that adopted in many models of galactic evolution; and stronger dependence of $\dot{\rho}_{*}$ on $\rho$ would seem implausible.

\section{COOLING FLOWS IN CLUSTER CORES}

A useful integral form for the star formation rate in a cooling flow is

$$
\dot{M}_{*}(r) \approx \frac{L_{x}}{0.5 \bar{H}+\lambda},
$$

where $L_{X}$ is the X-ray luminosity within a sphere of radius $r$. We correct the X-ray luminosity in the $0.5-3 \mathrm{keV}$ bandwidth over which most X-ray fluxes are observed to give the total emission at $k T \approx 3 \mathrm{keV}$, and we have adopted a characteristic value of $\alpha$ equal to 0.5 from equation (10). This solution only applies within the cooling radius, where the cooling time is less than a Hubble time, and we shall apply it to two of the best studied cooling flows.

In the case of NGC 1275, at the center of the Perseus cluster cooling flow, the cooling radius $r_{c}$ is $150 \mathrm{kpc}$ (Fabian et al. 1981). To estimate the X-ray luminosity within $r_{c}$, we note that the cluster X-ray luminosity out to $0.5 \mathrm{Mpc}$ over $0.5-3 \mathrm{keV}$ is $4 \times 10^{44} \mathrm{ergs} \mathrm{s}^{-1}$, with a core radius of $0.25 \mathrm{Mpc}$ (Jones and Forman 1984). Hence, adopting a temperature $k T=6.4 \mathrm{keV}$ and X-ray surface brightness profile proportional to $[1+$ $(r / a)]^{-1.3}$, the total X-ray luminosity within the cooling radius is $L_{X}\left(<r_{c}\right) \approx 1 \times 10^{44} \mathrm{ergs} \mathrm{s}^{-1}$. A check on this value comes from the estimate of "excess" X-ray luminosity, corrected downward by a factor of 2 according to the more accurate deconvolution technique of Henriksen (1985). Our formulation requires no a priori knowledge of the gravitational potential. 
Setting $\bar{T}(r)=5 \times 10^{7} \mathrm{~K}$ and taking $\lambda$ from equation (2), we infer

$$
\dot{M}_{*}=100(r / 75 \mathrm{kpc})^{5 / 4} M_{\odot} \mathrm{yr}^{-1} \quad(r \lesssim 75 \mathrm{kpc}) .
$$

Equation (12) is only likely to be valid at $r \lesssim 0.5 r_{c} \approx 75 \mathrm{kpc}$, the inferred star formation rate being $\dot{M}_{*} \approx 100 M_{\odot} \mathrm{yr}^{-1}$ at $r \lesssim 75 \mathrm{kpc}$. In the absence of heating, the inferred star formation rate would be higher by a factor $1+2 \lambda / \bar{H} \approx 1.8$.

Next consider the cooling flow around M87. The cooling radius is $\sim 17^{\prime}$ (or $\sim 70 \mathrm{kpc}$ ) (Stewart et al. 1984b). The enhanced X-ray flux out to $10 \mathrm{kpc}$ is $\sim 2 \times 10^{42} \mathrm{ergs} \mathrm{s}^{-1}$ (Schreier, Gorenstein, and Feigelson 1982), and $T=3.5 \times 10^{7} \mathrm{~K}$ so that we obtain

$$
\dot{M}_{*}=3.5(r / 35 \mathrm{kpc})^{0.6} M_{\odot} \mathrm{yr}^{-1} \quad(r<35 \mathrm{kpc}) .
$$

Again, this is only valid at $r \lesssim 0.5 r_{c} \approx 35 \mathrm{kpc}$. The reduction in star formation rate due to inclusion of heating is by a factor $\sim 2$ relative to the pure cooling model (Stewart et al. 1984b) at $\sim 30 \mathrm{kpc}$ and is substantially more at smaller radii.

Since most cooling flows have temperatures of several $\mathrm{keV}$, we expect that in general $\lambda \approx 0.5 \bar{H}$, so that supernova heating will reduce the required star formation rates by $\sim 50 \%$. These star formation rates are still very large for elliptical galaxies. However, it is possible to hide a considerable amount of star formation in the large volume encompassed by cluster cores without producing excessive surface brightness, and one should not necessarily regard star formation even with a normal IMF over such large scales as necessarily being an embarrassment. For example, in one of the best studied cluster cores, Coma, the search for diffuse intergalactic light has found a significant component of blue light, amounting to as much as $50 \%$ of the light from the galaxies (Thuan and Kormendy 1977). Of course, Coma is not presently the site of a cooling flow, but it may have been at an earlier epoch. It is tempting to identify this blue light with stars that formed in the accretion flow. A star formation rate as large as $\sim 100 M_{\odot} \mathrm{yr}^{-1}$ could have been sustained in this manner. The estimated supernova rates in current cooling flows pose more of a problem, as will be discussed below.

In general, the most stringent arguments that constrain the IMF in accretion flows have come from consideration of the colors of the central galaxies (Sarazin and O'Connell 1983). We shall present our own discussion of the colors of accreting galaxies, in the following section, but we mention here some specific comments pertaining to NGC 1275 and M87. In the bright central regions of NGC 1275 , the accretion rate is only a few tens of $M_{\odot} \mathrm{yr}^{-1}$, and is consistent with the observed rate of star formation (Wirth, Kenyon, and Hunter 1983). In the case of M87, the predicted star formation rate is consistent with the observed colors in the inner core. Gunn, Stryker, and Tinsley (1981) found that young stars, contributing $\sim 10 \%$ of the visual luminosity, provide a plausible, if not unique, interpretation of the UV spectrum of the central kiloparsec of M87, and infer a star formation rate of $\sim 0.2 M_{\odot} \mathrm{yr}^{-1} / 10^{10} L_{\odot}$. This is very similar to the estimated rate of mass loss from red giants, and this source could account for the observed colors if the ejected gas were efficiently converted into stars (Larson and Tinsley 1978). Hence there may be little need for an accretiondriven cooling flow in the M87 core. Further out, however, our mass inflow model implies that there is approximately a factor 10 more star formation continuing throughout M87 out to $\sim 50 \mathrm{kpc}$ than inferred from studying the central kiloparsec. Nevertheless, this is probably not inconsistent with the broad- band colors at large galactocentric distance even if a solar neighborhood IMF is adopted. For example, if the Gunn et al. estimate of the star formation rate applies to all the light observed out to $\sim 20 \mathrm{kpc}$, the inferred star formation rate would be $\sim 2 M_{\odot} \mathrm{yr}^{-1}$, comparable to the mass-flow rate inferred from our analysis of the cooling flow. Given the uncertainties in both contributions, one can only conclude that both effects are most likely contributing to the star formation rate and observed colors out to $\sim 20 \mathrm{kpc}$; further out, the accretion flow provides the dominant contribution to the local star formation rate.

\section{COOLING FLOWS IN ISOLATED ELLIPTICAL GALAXIES}

We turn finally to consider the hot gaseous coronae discovered to exist around elliptical galaxies outside the cores of rich clusters (Forman, Jones, and Tucker 1986). This phenomenon was predicted by Norman and Silk (1979) on the basis of radio observations and independently by Sarazin (1979), and these authors argued that ellipticals may retain most of the gas produced by evolving stars in extended hot coronae that are heated by supernova remnants. This halo gas must cool, albeit rather slowly, and will moreover be thermally unstable. The preceding results can now be applied to estimate the rate of gas accretion and accompanying star formation.

Typical values for the resolved elliptical galaxies in the Forman, Jones, and Tucker (1986) sample are $L_{X} \approx 15( \pm 10)$ $\times 10^{40}$ ergs s$^{-1}$. The enthalpy $H=3 \times 10^{15} T_{7}$, where $T_{7} \equiv$ $T / 10^{7} \mathrm{~K}$, and we infer that, since $T_{7} \approx 1$ for typical massive galaxy halos, supernova heating (see eq. [2]) may indeed regulate the mass flow. The inferred star formation rate from equation (11) is $\sim 0.5 M_{\odot} \mathrm{yr}^{-1}$. Over scales of several kiloparsecs, the observed star formation rates appear to about a factor $\sim 3$ less than rates obtained by taking the ratio of X-ray luminosity to enthalpy in isolated ellipticals (Nulsen, Stewart, and Fabian 1984). In the inner cores, the accretion flow is strongly suppressed by supernova heating, and a hydrostatic halo is present. We will now show that broad-band colors derived assuming an IMF appropriate to the solar neighborhood and containing high-mass stars are consistent with the observed colors of elliptical galaxies.

In order to compare these theoretical considerations with the properties of real galaxies, we used self-consistent stellar population synthesis models incorporating mass infall. These models were originally developed by Bruzual $(1981,1983)$ for investigations of galaxy evolution. A new feature here is the introduction of mass infall, which replenishes the material available for star formation. The models are defined by the IMF, star-formation rate (SFR), and the gas infall rate $(\dot{M})$, inplicitly assumed to be turned instantly into stars. Since at the present time little is known about the form of the IMF in elliptical galaxies and/or cooling flows, we use as a "minimal" assumption a time-invariant, Miller-Scalo (1979) IMF ${ }^{2}$ appropriate to the solar neighborhood, a lower stellar mass cutoff of $0.08 M_{\odot}$, and a variable upper mass cutoff $M_{\text {cut }}$, viz., $1,2,5,10$, or $25 M_{\odot}$. We also computed some models with a variable lower mass cutoff, e.g., only with stars more massive than $1 M_{\odot}$. Allowance for possible truncation of the IMF was investigated in order to compare our results with previous discussions (Sarazin and O'Connell 1983; Nulsen, Stewart, and

\footnotetext{
${ }^{2}$ Our adopted IMF is defined as a multiple power law, with the exponents ( $x \equiv 1$ power): $x=0.25$ for the mass range $0.08-1,1.01$ for $1-2,1.3$ (Salpeter slope) for 2-10, and 2-3 for 10-25 (in solar mass units).
} 
Fabian 1984). We have also tried models with the Salpeter $(x=1.3)$ IMF, and obtained qualitatively very similar results. The SFR as a function of time in the underlying galaxy is assumed to be exponential, with $e$-folding times of 0.83 and $1.44 \mathrm{Gyr}$ (Bruzual's $\mu=0.7$ and 0.5 models, respectively). This range of the $e$-folding times corresponds to the "best" estimates for spectral evolution of elliptical galaxies (Bruzual 1983; Djorgovski and Spinrad 1986). We start with an initial gas mass of $10^{11} M_{\odot}$, turn on the star formation and infall at $t=0$, and assume a range of infall rates from 0.01 to $100 M_{\odot}$ $\mathrm{yr}^{-1}$. Several tens of models spanning this range of parameters were computed.

Luminosity, colors, and supernova rates (SNR) are derived as functions of galaxy rest-frame time as the model evolves. We examine these quantities at the model age of $13 \mathrm{Gyr}$, which is a plausible estimate for the ages of early-type galaxies in our cosmic vicinity at the present epoch. The results change very little if a somewhat longer age is used (e.g., 16 Gyr) - the colors are just slightly redder, and the SNR is just slightly lower. The most serious drawback of these models is that they do not account for the metallicity differences between the galaxies: all colors are for solar abundance systems. This also means that we cannot straightforwardly reproduce (or account for) the well-known color-luminosity relations, which are driven mainly by the metallicity differences. The color-metallicity calibration of isochrones (e.g., Rabin 1982) should eventually allow an estimate of this effect. We computed colors in the ground-based photometric systems (e.g., UBVRIJKL), and the far-UV colors observable with the Hubble Space Telescope (HST). As may be intuitively expected, the bluer colors depend more strongly on the infall rates, and the HST data will provide much stronger constraints on possible infall rates than the presently available, ground-based photometry. We confine the present discussion to the $U-B$ and $B-V$ colors, as these are the only ones with published values for moderately large numbers of galaxies.

The range of the $\dot{M}$ rates adopted is quite adequate, as shown in Figure 1; at the low- $\dot{M}$ end, the infall is negligible, and the colors (at this age) are not perturbed by the additional star formation; at intermediate $\dot{M}$, the colors are bluer, as may be expected; and at the high- $\dot{M}$ end, we are practically in the steady state, constant-SFR regime, and the $U B V$ colors do not become any bluer with increasing $\dot{M}$ since the blue light is dominated by young stars. The curves with a higher average stellar mass are generally bluer, again as expected. The colors we obtain are in reasonable accord with those of the models calculated by Sarazin and O'Connell (1983) in the region of parameter space where we overlap, $M_{\text {cut }} \approx 2 M_{\odot}$. They did not investigate an IMF with massive stars.

The mass of the model galaxy changes as the new material is added. We find that luminosity and SNR are driven linearly by the infall rate as expected, for all values of $\dot{M}$ exceeding approximately $0.2 M_{\odot} \mathrm{yr}^{-1}$, or when the accreted mass exceeds $\sim 3 \%$ of that of the original galaxy (in the absence of any accretion). In order to compare the models with real galaxies, it is more convenient to express the quantities of interest as functions of $\dot{M}$ per unit total luminosity. As unit luminosity, we choose the amount corresponding to $M_{v}=-22$. At fixed luminosity, there must be limiting values of $\dot{M}$, colors, and supernova rates, which depend only very weakly on the IMF

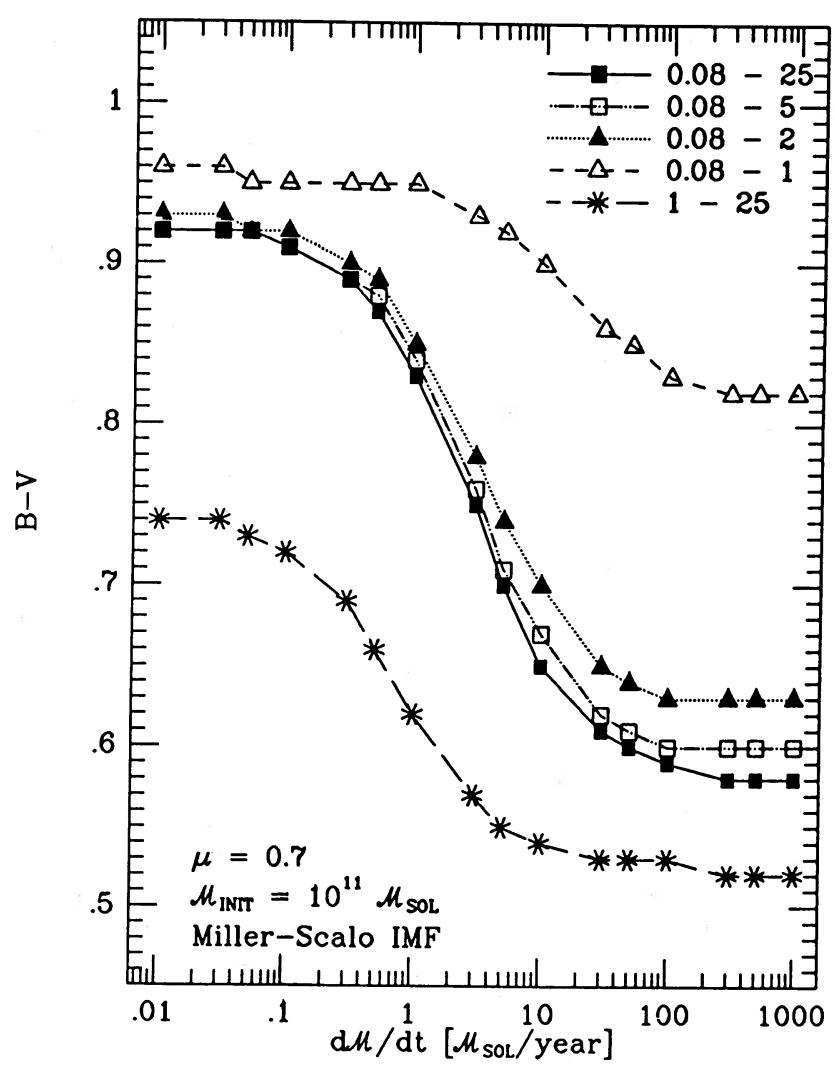

Fig. 1.- $U B V$ colors for the $\mu=0.7$ model galaxies at the age of $13 \mathrm{Gyr}$, as functions of infall rate. Starting galaxy mass is $10^{11} M_{\odot}$. Different symbols and lines correspond to different IMF mass ranges (in solar mass units). 
upper cutoff, although they do depend on the slope, and almost not at all on the SFR $e$-folding time of the underlying model, since this is practically a steady state SFR regime. This corresponds to a constant star formation rate dominated system. The limiting value of $\dot{M}$ for a $M_{V}=-22$ galaxy (or a galaxy slice of that luminosity) is $\sim 3 M_{\odot} \mathrm{yr}^{-1}$, or equivalently, constant star formation rate dominates the colors when $\dot{M}>10$ $M_{\odot} \mathrm{yr}^{-1}\left(10^{11} L_{\odot}\right)^{-1}$. For $M_{\text {cut }}=2 M_{\odot}$, the asymptotic colors are $(U-B)_{\mathrm{lim}}=0.34$ and $(B-V)_{\mathrm{lim}}=0.70$, whereas for $M_{\text {cut }}=25 M_{\odot}$, the corresponding values are 0.10 and 0.62 . The limiting supernova rates, again for $M_{V}=-22$, are $\sim 6-7$ per century for $M_{\text {cut }} \lesssim 10$, and 3 per century for $M_{\text {cut }}=5$ (Recall that the predicted colors in these models apply to solar metallicity systems only, and that abundance differences can modify these numbers appreciably.) The rates assume that every star with $M \gtrsim 5 M_{\odot}$ ends up as a supernova: this is almost certainly an overestimate.

As the data set, we use the $L_{X}$ measurements listed by Forman, Jones and Tucker (1985), and the $U B V$ photometry from the Second Reference Catalogue of Bright Galaxies (de Vaucouleurs, de Vaucouleurs, and Corwin 1976), the compilation of Longo and de Vaucouleurs (1983), and the measurements of Veron-Cetty (1984). This photometric data set is rather heterogeneous and leaves much space for improvement. Two desirable observational improvements would be to obtain high quality CCD surface photometry of these galaxies in a homogeneous $U B V R I$ photometric system, and far-UV spectroscopy, spectrophotometry, and imaging.

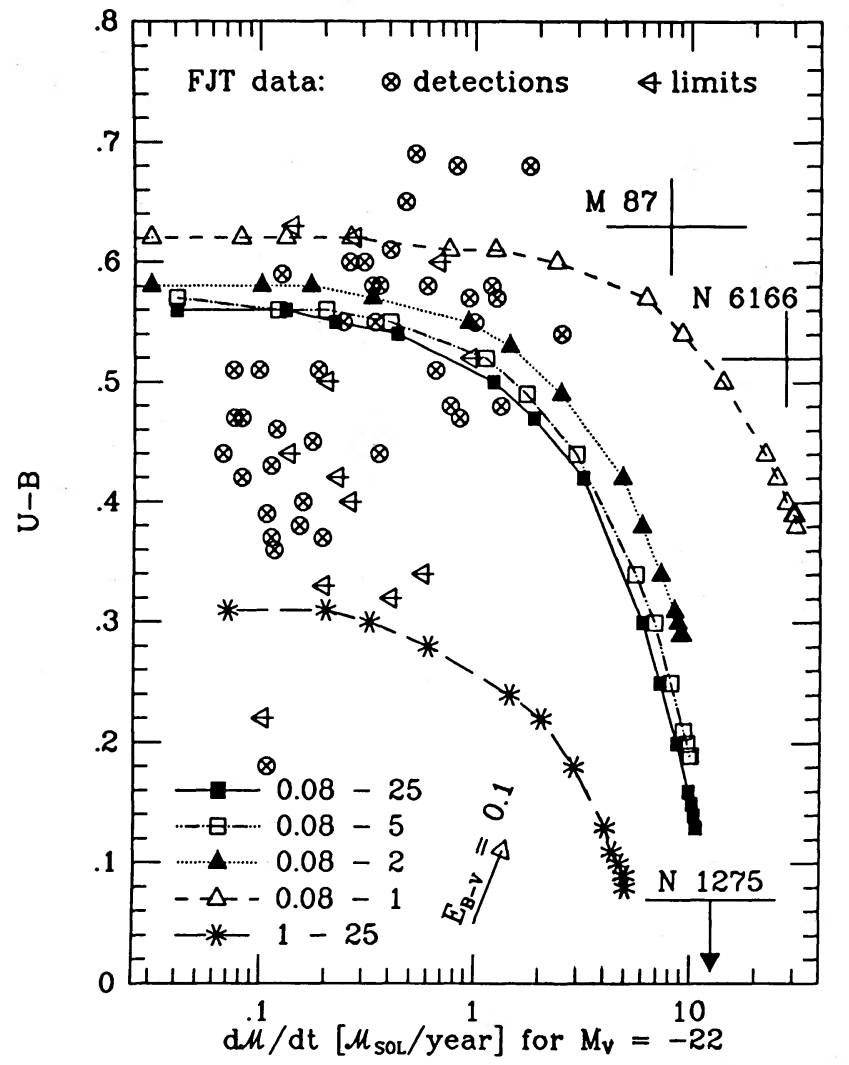
[11])

We compute the $\dot{M}$ rates according to the formula (see eq.

$$
\dot{M}=0.1 L_{40}\left(1+0.02 L_{40}^{1 / 2}\right)^{-1} M_{\odot} \mathrm{yr}^{-1}
$$

where $L_{40}=L_{X} / 10^{40}$ ergs $\mathrm{s}^{-1}$, and rescale them to $M_{V}=$ -22 , by using the galaxy $V$ magnitudes from these sources and redshifts given by Forman, Jones, and Tucker (1986) (we also assume $H_{0}=50 \mathrm{~km} \mathrm{~s}^{-1} \mathrm{Mpc}^{-1}$ for convenience). Equation (14) incorporates a correction factor that allows for the fact that the most luminous flows are usually hotter, and is consistent with the heating solution (11).

Even though the $\dot{M}$ values we obtain for elliptical galaxies do not differ substantially from previous discussions of cooling flows, we now demonstrate that the mass sink can consist of newly formed stars with a solar neighborhood IMF. We examine only models for galaxies within a narrow final mass range $(1-2) \times 10^{11} M_{\odot}$ and predict the colors for differing rates of mass infall. We have made a comparison with data points utilizing (14) and scaled to $M_{v}=-22$.

The results for the $\mu=0.7$ model are shown in Figure 2 . Most of the scatter in the observations is almost certainly due to the combination of abundance differences and random and systematic errors of the compiled photometry. The remaining scatter is probably due to differential reddening, which is not taken into account in the models. All that we can say is that the models and the data indicate a range of $0.1 \lesssim \dot{M} \lesssim 1 M_{\odot} \mathrm{yr}^{-1}$, somewhat lower than the values quoted by Nulsen, Stewart,

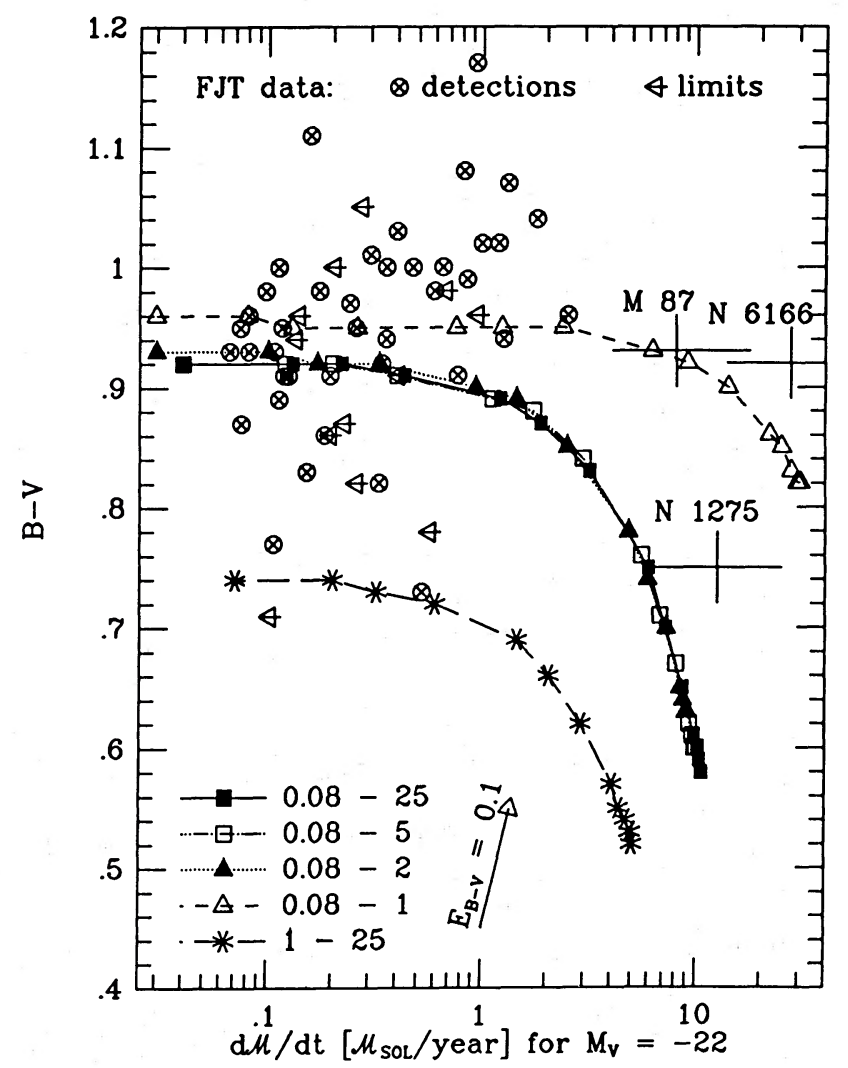

Fig. 2. $-U B V$ colors for the $\mu=0.7$ model galaxies at the age of $13 \mathrm{Gyr}$, as in Fig. 1, but with $\dot{M}$ rescaled to the unit luminosity, $M_{V}=-22$. For the data points, $\dot{M}$ values are evaluated from the $L_{X}$ measurements of Forman, Jones, and Tucker (1986), by using eq. (14); crossed circles correspond to $L_{X}$ detections, arrows to upper limits. Typical measurement errors for the colors are $\sim 0.03-0.05$. Inferred $\dot{M}$ and colors of some noted central cluster galaxies are marked with large crosses, whose sizes are only a rough indication of the measurement error bars. The meaning of model curves and symbols is as in Fig. 1. 
and Fabian (1984). The truncation of the IMF matters very little. This result largely dispels one of the biggest uncertainties for the present models and suggests that radically different forms of the IMF make little difference in the observed colors. The underlying galaxy dominates the colors for the moderate accretion flows present in elliptical galaxies.

The situation is somewhat different in the cluster cooling flows. For the "archetypical" cooling-flow galaxies M87, NGC 6166 , and NGC 1275 , we estimate $\dot{M}$ values of $\sim 10,90$, and $100 M_{\odot} \mathrm{yr}^{-1}$, and find that only the first two galaxies are too red relative to the models if a Miller-Scalo IMF is adopted. The situation is very similar for the $\mu=0.5$ model. NGC 1275 is in the asymptotic limit of the model curves, and its colors are insensitive to truncation. If a galaxy is any bluer, it must be so because of its low metal abundance or a presence of a nonthermal light source, e.g., associated with an active nucleus.

The inference that the very high values of $\dot{M}$ can be effectively hidden in the observed colors only if the IMF is modified as to lack massive stars is not very strong. This effect is clearly present at some level, but our models indicate that a reddening of $E_{B-V} \approx 0.1$ would make the truncated models indistinguishable from the Miller-Scalo IMF. One might argue that dust could not survive long in the hot X-ray gas, but $\mathrm{Hu}$ et al. (1986) find excess reddenings of this order are necessary to explain observations of cooling flows. In addition, Rabin's (1982) calibration of $B-V$ with metallicity suggests that an abundance of 3 times solar would correspond to a reddening of $0.3 \mathrm{mag}$, also sufficient to bring the models and data into agreement, although such high metallicities are only inferred for the underlying galaxy, the gas being about one-half solar metallicity. Our results are entirely consistent with those of Sarazin and
O'Connell (1983), whose models generally had $M_{\text {cut }}=1 M_{\odot}$. We have incorporated massive star formation and found that it makes little difference to colors in the visible spectrum for $\dot{M} \lesssim 10 M_{\odot} \mathrm{yr}^{-1}\left(10^{11} L_{\odot}\right)^{-1}$. The luminosity of the final galaxy as a function of $\dot{M}$ is shown in Figure 3. Our mass-tolight ratios are low $(\sim 2-6)$ in the asymptotic infall regime, but are readily augmented if a Salpeter type IMF were adopted. The standard Miller-Scalo model in the low $\dot{M}$ limit has $M / L=10$, appropriate to normal elliptical galaxies. Accretion flows persisting for more than $10^{10}$ yr have had ample time to make many red giants, and these tend to dominate the colors (Fig. 4).

Figure 5 shows a plot of the supernova rate (SNR) as a function of $\dot{M}$, both rescaled to an $M_{V}=-22$ galaxy (or a galaxy slice of that luminosity). The SNR is almost linearly dependent on $\dot{M}$, and it is thus driven almost by the infall rate alone. For example, in a giant, $M_{V}=-23$, galaxy, one expects a SNR of $\sim 30-80$ pcr century for an infall rate of $100 M_{\odot}$ $\mathrm{yr}^{-1}$. There are tentative indications of such a high supernova rate in NGC 1275, but the data based on photometric variations (Lyutj et al. 1977; Preston et al. 1974) need confirmation (Wirth, Kenyon, and Hunter 1983).

Are supernovae easily detectable at the distance of NGC 1275 ? With a distance modulus of $m-M=35$, a standard SNI with $M=-18.8$ at maximum should be visible in the outer parts of the galaxy. Its surface brightness is $\sim 19$ mag arcsec -2 with a seeing disk of $3^{\prime \prime}$, and it should stand out above the background light from the elliptical some $3 \mathrm{kpc}$ from the core. However, there is a legitimate worry as to whether such objects would form in the somewhat exotic conditions of the cooling flow. It is possible that intense star formation activity

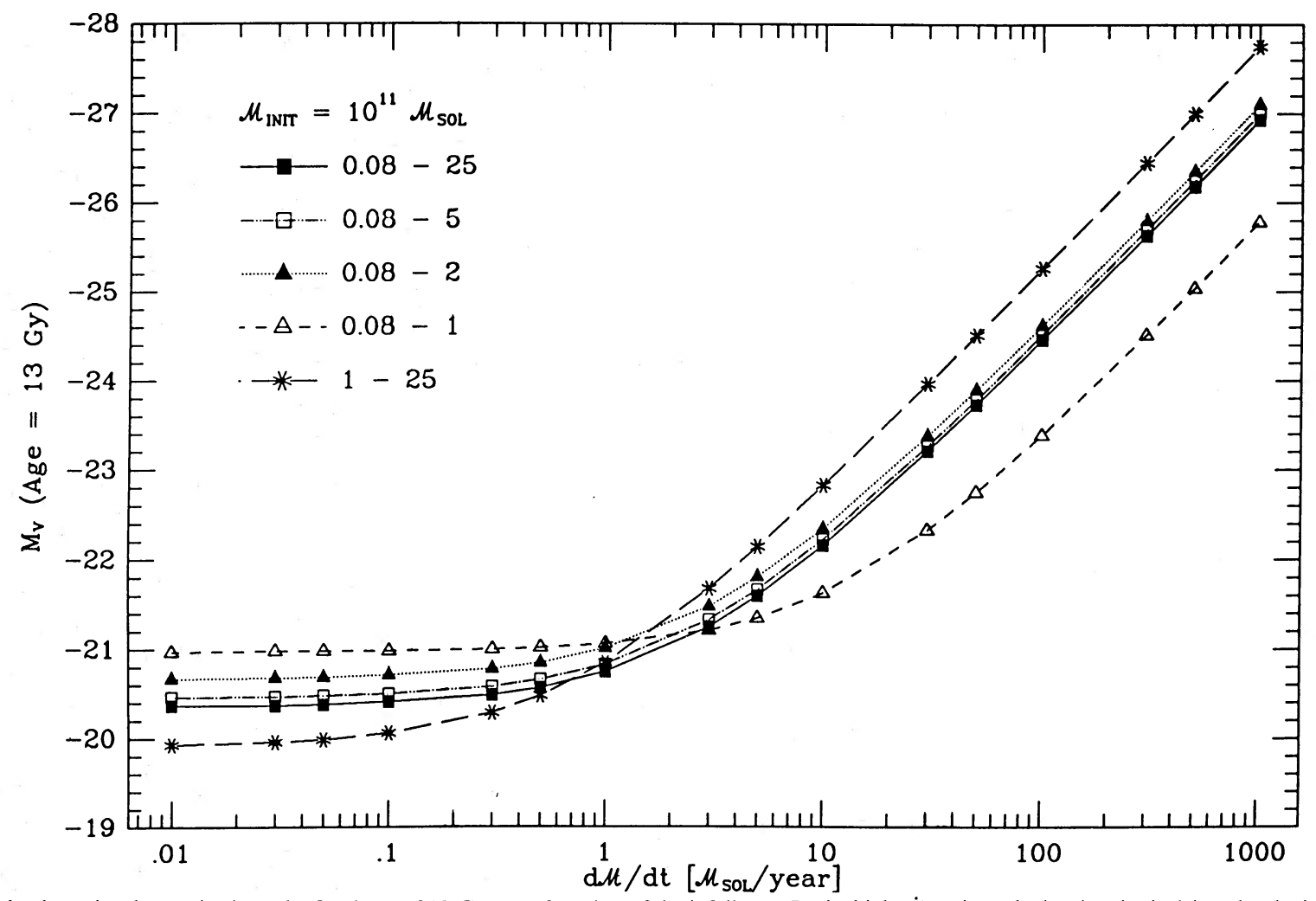

FIG. 3.-Absolute visual magnitude at the fixed age of $13 \mathrm{Gyr}$ as a function of the infall rate. In the high- $\dot{M}$ regime, the luminosity is driven by the infall rate in an almost linear manner. Different curves and symbols correspond to the different IMF cutoffs. 


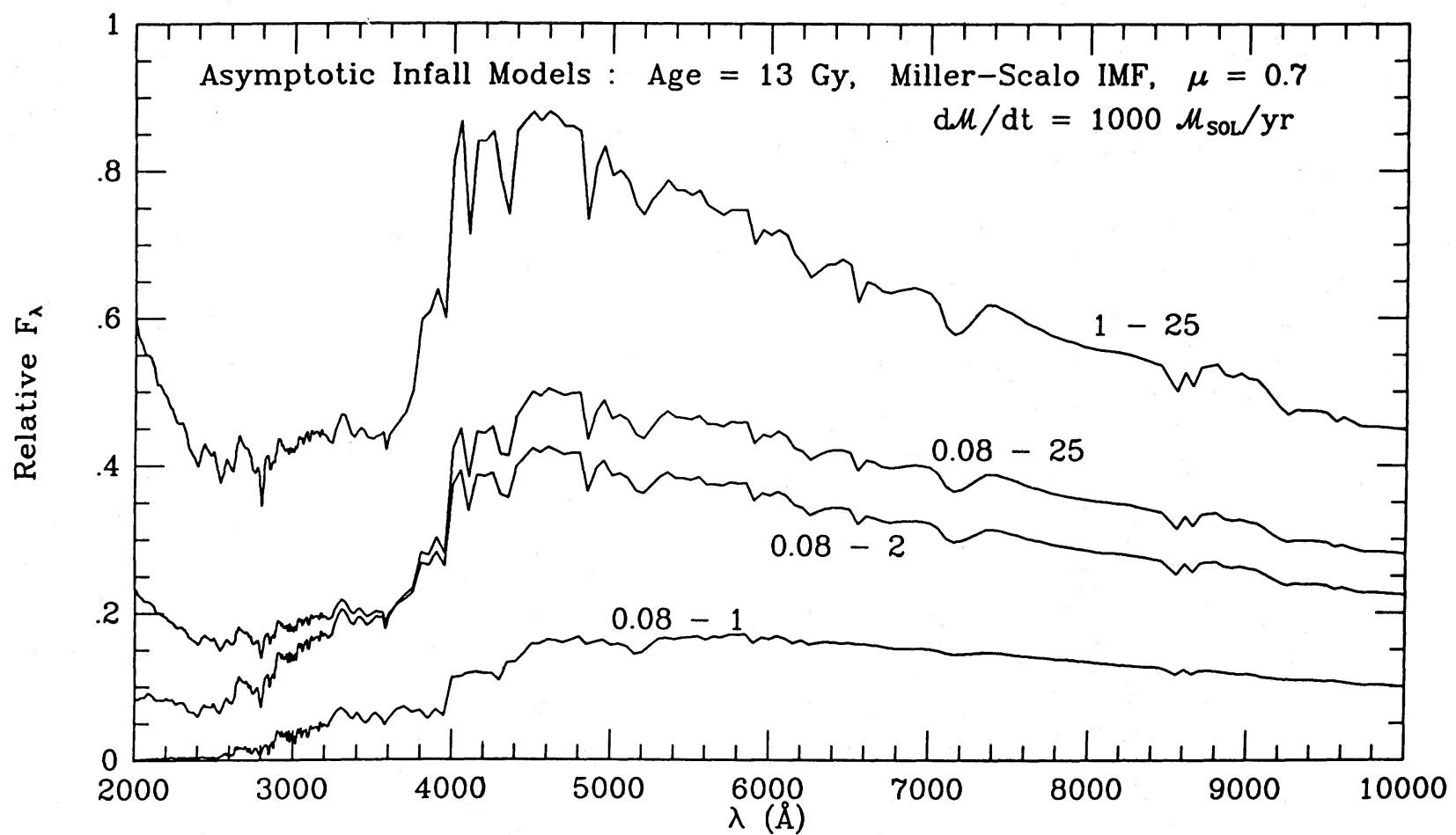

Fig. 4.-Synthetic spectra for models with different IMF mass ranges, in the asymptotic infall regime $\left(\dot{M}=1000 M_{\odot} \mathrm{yr}^{-1}\right.$ was used, but the results are practically identical with $\dot{M}=100 M_{\odot} \mathrm{yr}^{-1}$ ). All models with $M_{\text {cut }} \geq 2 M_{\odot}$ show some evidence of a young stellar population (Balmer lines).

favors faint supernovae that may be progenitors of remnants such as Cas A (Chevalier 1976). One recent example is the peculiar supernova found in the interacting galaxy NGC 4618 (Filippenko and Sargent 1985), models of which favor massive progenitors (Begelman and Sarazin 1986). The brightest radio supernova remnant in the starburst nucleus of M82 appears to have been decelerated at formation by a dense shell of progenitor ejecta (Kronberg, Bierman, and Schwab 1985) that would also have degraded much of its optical output.
Then, of course, there is the long-standing discrepancy between massive star death rates and pulsar and supernova remnant birth rates, on the one hand, and the rare optical supernovae discovered in the past thousand years in our own Galaxy, on the other hand. This discrepancy may be as much as an order of magnitude. Adopting the probable absolute magnitude at maximum of the peculiar supernova in NGC 4168, we infer that in NGC 1275 perhaps $90 \%$ of the predicted supernovae could be as faint as 19 th or 20 th magnitude at maximum light.

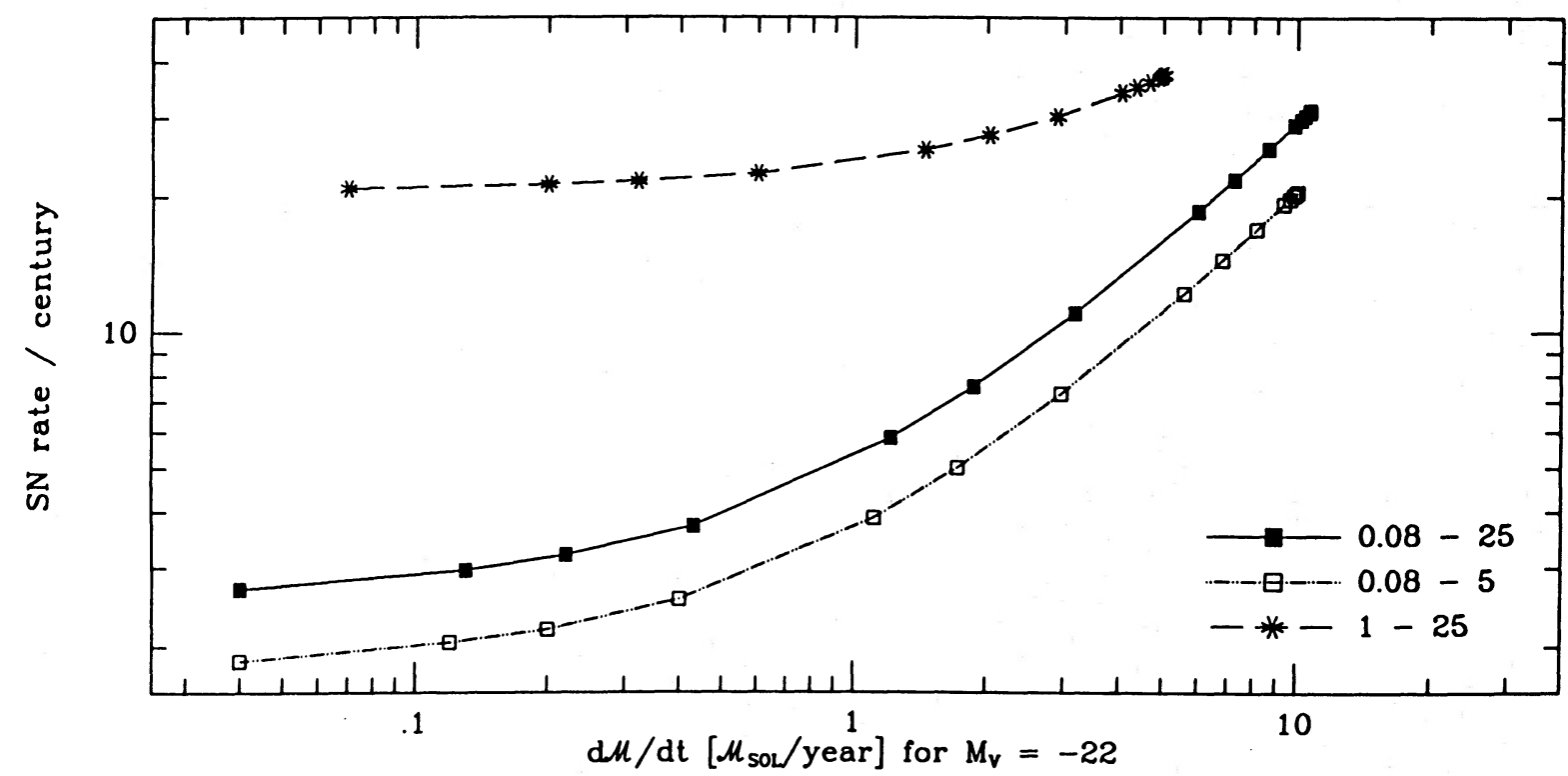

FIG. 5. - Supernova rates for the $\mu=0.7$ model galaxies at the age of $13 \mathrm{Gyr}$, with $\dot{M}$ rescaled to the unit luminosity. Different symbols and lines correspond to different IMFs. Models with $M_{\text {cut }}<2 M_{\odot}$ do not produce any supernovae. 
However, we anticipate that currently ongoing supernova searches should eventually be capable of providing a critical test of the infall models (McGraw, Angel, and Sargent 1980; Kare et al. 1981).

\section{SUPERNOVA HEATING AND OPTICAL FILAMENTS}

One assumption underlying the supernova heating model is that the energy input is evenly distributed throughout the accretion flow. The low-density cavity created by a supernova of energy $E$ exploding into a uniform medium survives for a time of order

$$
t_{M}=10^{5.5} E_{55}^{0.32} n_{2}^{-0.36} T_{7}^{-0.7} \mathrm{yr}
$$

until pressure equilibrium is attained, before being disrupted by the ambient gas. At this stage, its maximum radius is

$$
R_{M}=10^{1.86} E_{51}^{0.32} n_{2}^{0.36} T_{7}^{-0.2} \mathrm{pc} .
$$

The expansion remains adiabatic until a cooling radius is reached,

$$
R_{c}=10^{2.1} E_{51}^{0.29} n_{2}^{-0.41} \mathrm{pc},
$$

so that radiative losses become important, and a dense shell forms if $R_{c}<R_{M}$ or

$$
T_{7}<10^{-0.75} E_{51}^{0.15} n_{2}^{0.25} .
$$

The supernova remnants generally remain adiabatic in the massive halos of cluster cores, where $T_{7} \gtrsim 1$.

If the supernovae occur randomly at a rate proportional to the inferred star formation rate, all the energy remaining at maximum expansion will be effectively utilized in heating the accretion flow. This justifies our adopted heating rate $\lambda=v E$. The remnant volume filling factor $Q$ provides an indication of the inhomogeneity of the inflow. The cooling gas is thermally unstable, and the time scale for ensuing stellar formation and $\mathrm{SN}$ heating, $t_{M}$, is short compared to the cooling time. The SN generated inhomogeneities could be the sources of the observed $\mathrm{H} \alpha$ filaments, probably serving as the local sites of enhanced thermal instability and cooling. We estimate that

$$
Q=\frac{4 \pi}{3} v \dot{\rho}_{*} R^{3} r_{100}^{-3} n_{3}^{1.44} T_{7}^{1.3} \dot{M}_{100}
$$

where $r_{100}=r / 100 \mathrm{kpc}, n_{3}=n / 10^{-3} \mathrm{~cm}^{-3}, T_{7}=T / 10^{7} \mathrm{~K}$, and $\dot{M}_{100}=M / 100 M_{\odot} \mathrm{yr}^{-1}$ denote parameters scaled to values that may be appropriate near the cooling radius.

If gravitational instability occurs in the cooling filaments, it must occur over a time scale that is comparable to or less than $t_{M}$. With $Q \approx 10^{-4}$, this means that $\sim 10^{8} M_{\odot}$ of gas could be unstable to star formation over $\sim 10^{6} \mathrm{yr}$ at any given instant in a cooling flow. During the ensuing cooling, this gas will radiate strongly in the visible, and a $100 M_{\odot} \mathrm{yr}^{-1}$ flow will radiate the equivalent of the kinetic energy of about one supernova per yr, or $\sim 3 \times 10^{43} \mathrm{ergs} \mathrm{s}^{-1}$ in line radiation. This is in reasonable accord with observations of $\mathrm{H} \alpha$ emission from filaments in cooling flows. In particular, the high $\mathrm{H} \alpha$ luminosity and surface brightness of the filament complex around NGC 1275 (Cowie et al. 1983) offers considerable support for a local heat source such as the one advocated here. The observed filament line ratios are consistent with a shock-heating model $(\mathrm{Hu}$, Cowie, and Wang 1985). While the imaged $\mathrm{H} \alpha$ observations in this latter study of cooling filaments should, in principle, provide an independent estimate of $\dot{M}$, there are considerable uncertainties in inferring both the total $\mathrm{H} \alpha$ flux from long-slit spectroscopy and in the number of $\mathrm{H} \alpha$ photons produced per hydrogen ionization.

$\mathrm{Hu}$, Cowie, and Wang (1985) raise one additional objection to normal star formation in cooling flows. The optical emission filaments are generally confined to regions well inside the cooling flow. The X-ray deconvolution models suggest that mass deposition from the flow occurs at radii $(\gtrsim 50 \mathrm{kpc})$ where there is little, if any, optical evidence for cooling gas. A simple resolution of this apparent contradiction may come about as follows. Our models naively assume that cooling and star formation were instantaneous. In fact, there must be a time-delay due to the finite cooling time in the flow. Knowing $\dot{M}$ and $n$, we can estimate that the flow velocity is of order

$$
\begin{aligned}
& v=\dot{M}\left(4 \pi \rho r^{2}\right)^{-1}=4\left(\dot{M} / 100 M_{\odot} \mathrm{yr}^{-1}\right) \\
& \times\left(n / 0.01 \mathrm{~cm}^{-3}\right)(75 \mathrm{kpc} / r)^{2} \mathrm{~km} \mathrm{~s}^{-1}
\end{aligned}
$$

The long cooling time ensures that the $\mathrm{X}$-ray emitting gas could have moved a distance of order $50 \mathrm{kpc}$ before cooling to a low enough temperature to produce most of the $\mathrm{H} \alpha$ filaments in the inner regions.

A further complicating factor is that the cooling flow is thermally unstable and forms filaments, which are then in our model followed by massive star formation and further supernova remnant-induced filament formation. However, the radial density and temperature gradients in the underlying flow should ensure that the filament formation and associated emission are biased toward the central regions of the cooling flow. Finally, we note that the uncertainties in the radial profiles of density, temperature, star formation rate, enthalpy, and gravitational potential may be such that a model with constant $\dot{M}$ independent of radius outside $\sim 20 \mathrm{kpc}$ cannot be excluded.

NGC 1275 has A-type stellar features in the spectrum, and this has led Sarazin and O'Connell (1983) to advocate an IMF in the accretion flow with an upper mass cutoff near $3 M_{\odot}$. Figure 4 clearly shows that A-type features occur in the predicted spectrum for a range of values of $M_{\text {cut }}>2 M_{\odot}$. However, the predicted spectrum after a Hubble time is far too red to contain predominantly A stars: the observed spectrum resembles rather a recent accretion flow that has only persisted for several billion years, thereby avoiding much red giant formation. In Figure 6, we show the integrated spectrum for the asymptotic infall regime at various ages. We interpret the NGC 1275 spectrum as being consistent with a star formation rate of $\sim 100 M_{\odot} \mathrm{yr}^{-1}$ that is relatively recent ( $\lesssim 3 \mathrm{Gyr}$ ), and with no restriction necessary on the upper mass cut-off. Support for such a high star formation rate including massive stars is presented by Wirth, Kenyon, and Hunter (1983), who find that some $20 \%$ of the light in the inner $10 \mathrm{kpc}$ is contributed by $B$ stars. Population synthesis of their absorption line spectra provided evidence for a star formation rate of $\sim 30 M_{\odot}$ $\mathrm{yr}^{-1}$ in this region. The only way to produce a young population (B and A type) dominated spectrum is if the flows are young, certainly less than 3-5 Gyr (Fig. 6).

The extended nonthermal radio emission around cooling flow galaxies provides additional evidence of old supernova remnant activity associated with ongoing star formation. In our own Galaxy, the nonthermal radio power $\left(\sim 4 \times 10^{38}\right.$ ergs $\left.s^{-1}\right)$ is mostly due to relativistic electrons accelerated in shocks driven by old remnants, the total energy input from which is $\sim 3 \times 10^{41}-10^{42} \mathrm{ergs} \mathrm{s}^{-1}$. In the intracluster medium, the supernova remnants injected from galaxies suffer additional loss of kinetic energy due to adiabatic expansion: with a 


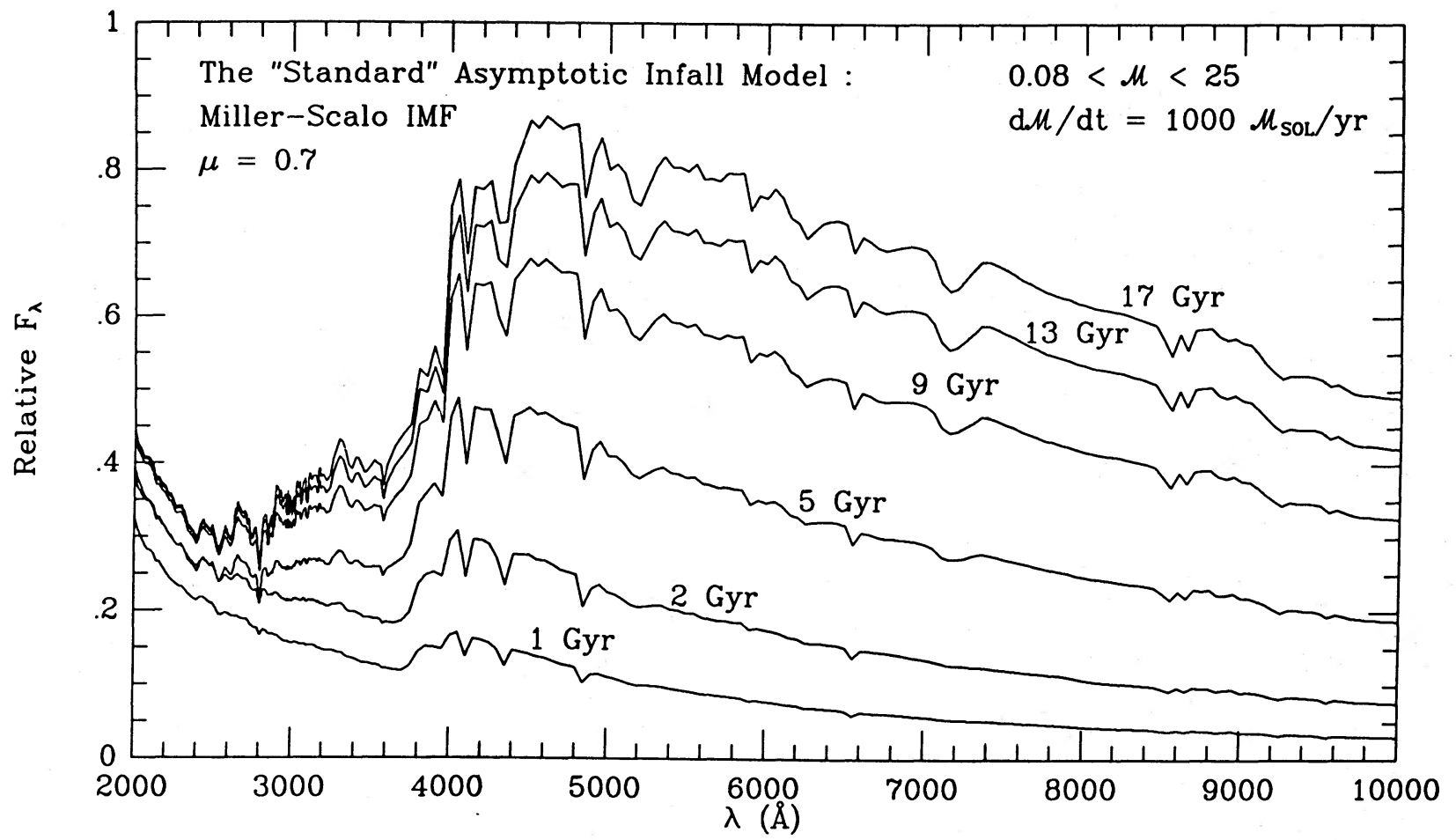

Fig. 6.-Synthetic spectra for the model with the complete Miller-Scalo IMF, as it evolves in time. The asymptotic infall regime $\left(\dot{M}=1000 M_{\odot} \mathrm{yr}^{-1}\right)$ was used, but the results are practically identical with $\dot{M}=100 M_{\odot} \mathrm{yr}^{-1}$ case. Only the very young models (a few Gyr age) are dominated by the young $(\mathrm{O}, \mathrm{B}, \mathrm{A})$ stars - red giants and dwarfs dominate the light at later ages.

mean separation between galaxies of $\sim 0.4 \mathrm{Mpc}$, the loss of kinetic energy of the remnants relative to adiabatic losses by the relativistic electrons amounts to a factor of $\sim 0.2$ $\mathrm{Mpc} / R_{\text {galaxy }} \approx 20$. Hence scaling relative to our own Galaxy, the intracluster radio source is more efficient, and a diffuse radio source of $10^{42}$ ergs $\mathrm{s}^{-1}$, as seen around NGC 1275, requires a supernova energy injection rate of $\sim 3 \times 10^{43}-10^{44}$ ergs $s^{-1}$. This lies in the same range as the supernova injection rate inferred from the star formation rate associated with the cooling flow.

Another observational consequence of supernova heating may be the strong correlation observed for many galaxies between X-ray and optical luminosities (Long and Van Speybroeck 1983). Since the supernovae probably fail to drive a wind, the energy available per unit mass of gas is given simply by the depth of the galaxy potential well, and one therefore expects that $L_{X} \propto M \sigma^{2} \propto L_{V}^{3 / 2}$, where $M$ is the galaxy mass, $L_{V}$ is its visual luminosity, $\sigma$ its mean velocity dispersion, and we have taken $M \propto L_{V} \propto \sigma^{4}$ (Nulsen, Stewart, and Fabian 1984).

\section{SUMMARY}

Mass accretion rates inferred from cooling flows in rich cluster cores may be overestimated if gas heating associated with the inferred star formation is neglected. That star formation must be occurring seem inevitable, given the observed cooling rates. Massive star formation may be suppressed, but we find the evidence from central galaxy colors to be not overwhelming even in the best studied examples in view of uncertain corrections to model predictions due to reddening by dust and the effect of enhanced metallicities relative to the Sun which are not incorporated into our models. One lacks adequate photometry to make a convincing case: ultraviolet observations should greatly improve this situation. Certainly, massive stars are forming in the cooling flows centered on NGC 1275 , and this flow must, moreover, be young ( $\$ 3 \mathrm{Gyr}$ ).

We have suggested that there is optical evidence for the existence of fossil cooling flows in clusters (e.g., Coma), where there is certainly a negligible cooling flow at present, and for young, recently initiated cooling flows (e.g., around NGC 1275 in Perseus), where the flow apparently began 1-3 Gyr ago. What physical mechanism could be responsible for producing short-lived cooling flows lasting for perhaps only $1 / 10$ of the Hubble time? Two obvious candidates are thermal conductivity and dynamical evolution. The role of thermal conductivity is globally sensitive to the gas temperature profile (Binney and Cowie 1975; Bertschinger and Meiksin 1986); indeed, it efficiently suppresses cooling if the revised cluster gas temperatures of Henricksen $(T \approx 10-15 \mathrm{keV})$ are generally applicable. Dynamical evolution as predicted in the cold dark matter scenario of galaxy clustering suggests that many clusters are evolving today on a time-scale as short as $1 \mathrm{Gyr}$ (Davis et al. 1985). Morphological studies of rich X-ray emitting clusters confirm that many are still undergoing dynamical relaxation (Beers, Geller, and Huchra 1982). Since a cooling flow only occurs over a relatively narrow window in the $(n, T)$ plane, either of these processes might conspire to restrict the effective duration of the cooling flows.

In summary, we have found that the assumption of a solar neighborhood initial mass function is entirely adequate for accounting for the observed colors of elliptical galaxies surrounded by extended gaseous coronae. Even in the cluster cores, the evidence for an IMF with exclusively low mass stars is inconclusive. There are at least two potential tests of our conclusion that exclusively low mass star formation $\left(M_{\text {cut }}<2\right.$ 
$\left.M_{\odot}\right)$ may be unnecessary in cooling flows. The ultraviolet spectrum of the central galaxy is very sensitive to $M_{\text {cut }}$. Moreover, the supernova rate is high if a Miller-Scalo IMF is adopted. A SNI rate of $\sim 1$ per yr per $100 M_{\odot} \mathrm{yr}^{-1}$ should be detectable if supernova searches were to focus on cooling flow galaxies.
We thank Ed Bertschinger and Paul Nulsen for several helpful comments on an earlier draft of this paper. This research has been supported in part by NASA under grant NGR 05-003-578 and by Cal Space.
Beers, T. C., Geller, M. J., and Huchra, J. P. 1982, Ap. J., 257, 23.

Begelman, M. C., and Sarazin, C. 1985, preprint.

Bertschinger, E., and Meiksin, A. 1986, preprint

Branduardi-Raymont, G., Fabricant, D., Feigelson, E., Gorenstein, P., Grind-

lay, J., Soltan, A., and Zamorani, G. 1981, Ap. J., 248, 55.

Bruzual, A., G. 1981, Ph.D. thesis, University of California at Berkeley. .1983, Ap. J., 273, 105.

Cowie, L. L., Hu, E. M., Jenkins, E., and York, D. 1983, Ap. J., 272, 29.

Davis, M., Efstathiou, G., Frenck, C., and White, S. D. M. 1985, Ap. J., 292, 371 .

de Vaucouleurs, G., de Vaucouleurs, A., and Corwin, H. G. 1976, Second Reference Catalogue of Bright Galaxies (Austin: University of Texas Press).

Djorgovski, S., and Spinrad, H. 1986, $A p$. J., in press.

Fabian, A. C., Hu, E. M., Cowie, L. L., and Grindlay, J. 1981, Ap. J., 248, 47.

Fabian, A. C., Nulsen, P. E. J., and Cqnizares, C. R. 1982, M.N.R.A.S., 201, 933. . 1984, Nature, 310, 733.

Fabricant, J., and Gorenstein, P. 1983, Ap. J., 267, 535.

Filippenko, A. V., and Sargent, W. L. W. 1985, Nature, 316, 407.

Forman, W., Jones, C., and Tucker, W. 1986, Ap. J., 301, 836.

Gilmore, G., and Arnaud, K. A. 1986, to be published.

Gunn, J. E., Stryker, L. L., and Tinsley, B. M. 1981, Ap. J., 249, 48.

Helou, G., Soifer, B. T., and Rowan-Robinson, M. 1985, Ap. J. (Letters), 298 L7.

Henriksen, M. J. 1985, NASA Goddard preprint LHEA 85-602.

Hu, E. M., Blades, C., Cowie, L. L., and Machetto, D. 1986, in preparation.

Hu, E. M., Cowie, L. L., and Wang, Z. 1985, Ap. J. Suppl., 59, 447.

Jones, C., and Forman, W. 1984, Ap. J., 276, 38.

Jura, M. 1977, Ap. J., 212, 634.

Kare, J. T., Pennypacker, C. R., Buller, R. A., Mast, T. S., Crawford, F. S., and Burns, M. S. 1981, in Supernovae: A Survey of Current Research, ed. M. J. Rees and R. J. Stoneham (Dordrecht: Reidel), p. 325.

Klein, R. J., Sandford, M. T., and Whitaker, R. W. 1983, Ap. J. (Letters), 271, L69.

Kronberg, P. P., Biermann, P., and Schwab, F. R. 1985, Ap. J., 291, 693.

Larson, T., and Tinsley, B. M. 1978, Ap. J., 210, 46.
REFERENCES

Long, K. S., and Van Speybroeck, L. 1983, in Accretion Driven Stellar X-Ray Sources, ed. W. H. G. Lewin, and E. P. J. van der Heuvel (Cambridge: Cambridge University Press).

Longo, G., and de Vaucouleurs, A. 1983, Univ. Texas Monog. Astr., No. 3.

Lyutj, V. M., Penston, M. J., Selmes, R. A., Becklin, E. E., and Neugebauer, G. 1976, M.N.R.A.S., 169, 357.

McGraw, J. T., Angel, J. R. P., and Sargent, T. A. 1980, Proc. SPIE, 264, 20.

McKee, C. M., and Ostriker, J. P. 1977, Ap. J., 218, 148.

Miller, G. E., and Scalo, J. M. 1979, Ap. J. Suppl., 41, 513

Mushotzky, R. 1984, Proc. Conf. Nonthermal Very High Temperature Phenomena in $X$-Ray Astronomy in press.

Norman, C., and Silk, J. 1979, Ap. J. (Letters), 233, L1.

Nulsen, P. E. J., Stewart, G. C., and Fabian, A. C. 1984, M.N.R.A.S., 208, 185.

Oemler, G., and Tinsley, B. M. 1979, A.J., 84, 985.

Rabin, D. 1982, Ap. J., 261, 85.

Romanishin, W. 1986, preprint.

Rose, J. A. 1985, A.J., 90, 1927.

Rothenflung, R., and Arnaud, M. 1985, Astr. Ap., 144, 431.

Sarazin, C. L. 1979, Ap. J. (Letters), 20, 93.

. 1985, Rev. Mod. Phys., in press.

Sarazin, C. L., and O'Connell, R. W. 1983, Ap. J., 268, 557.

Scalo, J. 1985, preprint.

Schreier, E. J., Gorenstein, P., and Feigelson, E. P. 1982, Ap. J., 261, 42

Silk, J. 1985, Ap. J., 297, 1.

Stewart, G. C., Canizares, C. R., Fabian, A. C., and Nulsen, P. E. J. 1984b, Ap. $J ., 278,536$.

Stewart, G. C., Fabian, A. C., Jones, C., and Forman, W. 1984a, Ap. J., 285, 1.

Thuan, T. X., and Kormendy, J. 1977, Pub. A.S.P., 89, 466.

Tucker, W. H., and Rosner, R. 1983, Ap. J., 267, 547.

van Breugel, W., Filippenko, A. V., Heckman, T., and Miley, G. 1985, Ap. J., 293, 83 .

Veron-Cetty, M. P. 1984, ESO preprint No. 330.

White, R. E., and Chevalier, A. C. 1984, Ap. J., 280, 561.

Wirth, A., Kenyon, S. J., and Hunter, D. A. 1983, Ap. J., 269, 102.

Yahil, A., and Ostriker, J. P. 1973, Ap. J., 185, 787.

G. BRuzual A.: Centro de Investigaciones de Astronomia, Merida, Venezuela

S. DJoRgovski: Center for Astrophysics, Harvard University, 60 Garden Street, Cambridge, MA 02138

J. SILK and R. WYSE: Astronomy Department, University of California, Berkeley, CA 94720 